\title{
PIEZOELECTRIC BEAM FINITE ELEMENT MODEL AND ITS REDUCTION AND CONTROL
}

\author{
KUTIŠ Vladimír ${ }^{*}$, PAULECH Juraj ${ }^{1}$, GÁLIK Gálik ${ }^{1}$, MURÍN Justín ${ }^{1}$ \\ ${ }^{1}$ Slovak University of Technology in Bratislava, Faculty of Electrical Engineering and Information Technology, \\ Institute of Automotive Mechatronics, Ilkovičova 3, 81219 Bratislava, Slovakia, \\ e-mail:vladimir.kutis@stuba.sk
}

\begin{abstract}
The paper deals with the development of the finite element method (FEM) model of piezoelectric beam elements, where the piezoelectric layers are located on the outer surfaces of the beam core, which is made of functionally graded material. The created FEM model of piezoelectric beam structure is reduced using the modal truncation method, which is one of model order reduction (MOR) method. The results obtain from reduced state-space model are compared with results obtain from finite element model. MOR state-space model is also used in the design of the linear quadratic regulator (LQR). Created reduced state-space model with feedback with the LQR controller is analysed and compared with the results from FEM model.
\end{abstract}

KEYWORDS: Finite Element Method, Piezoelectric Analysis, State-Space Model, LQR Control, MOR

\section{Introduction}

Smart materials are a very important building blocks in mechatronic applications [1]. These materials include shape memory materials, magnetorheological, functionally graded, piezoelectric, and other types of modern materials [2]. Their main feature is their possibility to extend the functional usage of a given system, to modify the dynamic behaviour of a given system or to allow a return to the original state of the system under certain conditions.

Frequently used smart materials are piezoelectric materials, which couple the mechanical and electrostatic fields. This physical coupling predetermines piezoelectric materials for use as sensors or actuators [3]. They allow the mechanical deformation of piezoelectric material caused by external forces to be converted to an electric potential on outer surfaces of piezoelectric material and vice versa electrical potential on outer surfaces of piezoelectric material caused by external loading to be converted to a mechanical deformation of piezoelectric material. Such use of piezoelectric materials in mechatronic systems is closely connected to control systems and controller design according to defined requirements. In mechatronic systems the so-called modern control is frequently used [4]. State-space model of a given physical system is used in the design of controllers using modern control theory. From a control viewpoint, it is very desirable that the state-space model is not too large. The finite element method (FEM) [5] is most often used for the physical description of a given system, but the FEM model is dimensionally significantly larger than the state-space model suitable for control purposes. It is therefore necessary to reduce the size of FEM model and this process is called model order reduction (MOR) [6]. Reduced state-space model based on detailed FEM model can be used to design of linear quadratic controller (LQR) [7].

This paper deals with the development of the FEM model of piezoelectric beam element, where the piezoelectric layers are located on the outer surfaces of the beam core, which is made of functionally graded material (FGM) [8,9]. Connection of FGM beam with piezoelectric layers can be considered as suitable smart material composition for mechatronic applications. Subsequently, the FEM model of a system is transformed and reduced to state- 
space model by modal truncation method, which is one of the MOR methods [6]. Created state-space model is used to build feedback gain matrix and closed loop reduced state-space model is analysed. Developed FEM model, MOR technique, state-space model and LQR control are implemented in FEM code MultiFEM, which is programmed in software Mathematica [10].

\section{Homogenized material properties of beam}

Let us consider straight sandwich beam with core made from functionally graded material (FGM) and top and bottom layers made from piezoelectric material with constant material properties - Fig. 1. Cross-section of FGM core has height $h_{F G M}$ and depth $b$, one piezoelectric layer has height $h_{p}$ and depth $b$. Cross-section area of FGM core is $A_{F G M}$, quadratic moment of inertia of FGM core to neutral axis of core is $I_{F G M}$, cross-section area of one piezoelectric layer is $A_{p}$ and quadratic moment of inertia of piezolayer to its neutral axis is $I_{p}$.

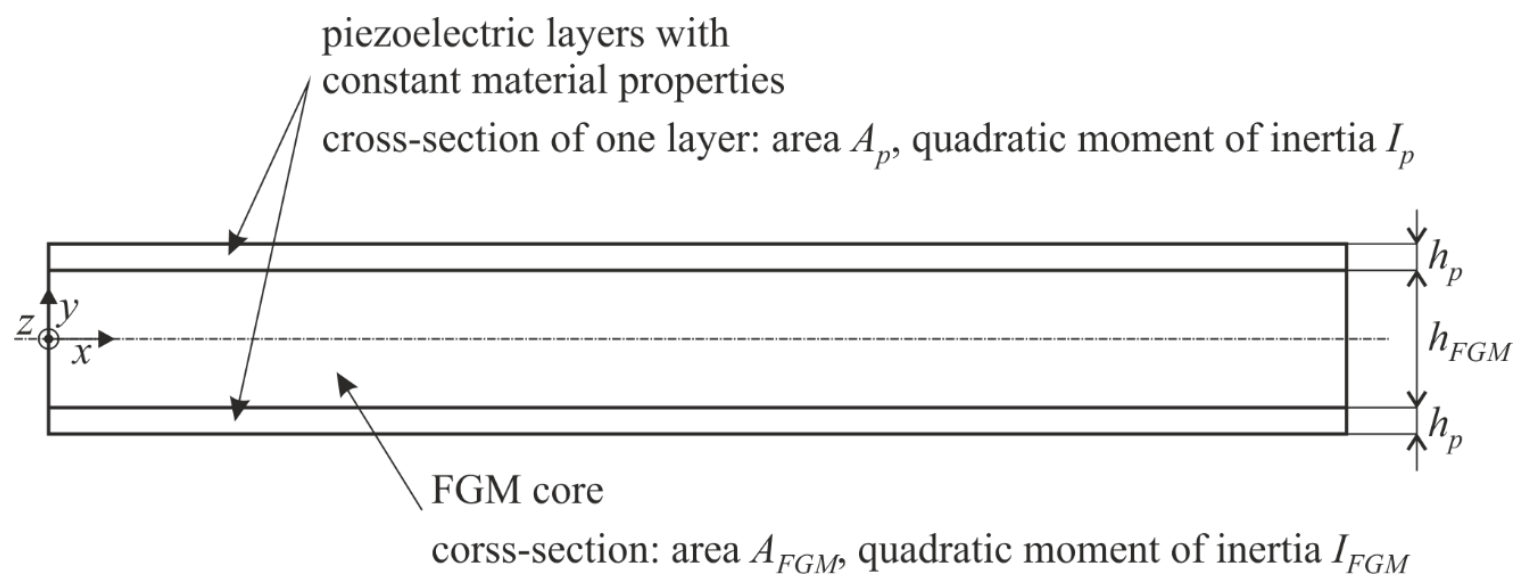

Fig. 1 Beam made of FGM core and piezoelectric layers.

The composite material of the FGM core arises by mixing two components - matrix and fibers, that are approximately of the same geometrical form and dimensions.

Both the fiber volume fraction $v_{f}(x, y)$ and matrix volume fraction $v_{m}(x, y)$ are chosen as a polynomial function of longitudinal position $x$, and with continuous and symmetrical variation through its height $h_{F G M}$ with respect to the neutral plane of the FGM core. The volume fractions are assumed to be constant through the cross-section depth $b$. At each point of the FGM core it holds: $v_{f}(x, y)+v_{m}(x, y)=1$.

Young modulus of the constituents, i.e., fibers $-E_{f}(x, y)$ and matrix $-E_{m}(x, y)$, can analogically vary as it is stated by the variation of volume fractions. For effective Young's modulus $E_{F G M}(x, y)$ and effective density of FGM core we can write [11]

$$
\begin{aligned}
& E_{F G M}(x, y)=v_{f}(x, y) E_{f}(x, y)+v_{m}(x, y) E_{m}(x, y) \\
& \rho_{F G M}(x, y)=v_{f}(x, y) \rho_{f}(x, y)+v_{m}(x, y) \rho_{m}(x, y)
\end{aligned}
$$

\subsection{Homogenized properties of FGM core}

Homogenized Young's modulus for axial loading $E_{F G M}^{H N}(x)$, bending $E_{F G M}^{H M}(x)$ and homogenized density $\rho_{F G M}(x)$ of FGM core with cross-section $A_{F G M}$ (height $h_{F G M}$ and depth $b)$ and quadratic moment of inertia $I_{F G M}$ can be expressed in form 


$$
\begin{aligned}
E_{F G M}^{H N}(x) & =\frac{\int_{-h_{F G M} / 2}^{h_{F G M} / 2} b E_{F G M}(x, y) d y}{A_{F G M}} \\
E_{F G M}^{H M}(x) & =\frac{\int_{-h_{F G M} / 2}^{h_{F G M} / 2} b y^{2} E_{F G M}(x, y) d y}{I_{F G M}} \\
\rho_{F G M}(x) & =\frac{\int_{-h_{F G M} / 2}^{h_{F G M} / 2} b \rho_{F G M}(x, y) d y}{A_{F G M}}
\end{aligned}
$$

Homogenized Youngs' moduli and density of FGM core are dependent only on axial location $x$ and not on transversal location $y$.

\subsection{Homogenized properties of beam with FGM core and piezoelectric layers}

Homogenized Young's modulus for axial loading $E^{H N}(x)$, bending $E^{H N}(x)$ and homogenized density $\rho(x)$ of beam with FGM core and piezoelectric layers can be expressed in form

$$
\begin{gathered}
E^{H N}(x)=\frac{A_{F G M}}{A} E_{F G M}^{H N}(x)+\frac{2 A_{p}}{A} E_{p} \\
E^{H M}(x)=\frac{I_{F G M}}{I} E_{F G M}^{H N}(x)+\frac{2 I_{p z}}{I} E_{p} \\
\rho(x)=\frac{A_{F G M}}{A} \rho_{F G M}(x)+\frac{2 A_{p}}{A} \rho_{p}
\end{gathered}
$$

where $A$ is total cross section $\left(A=A_{F G M}+2 A_{p}\right), I$ is total quadratic moment of inertia and $I_{p z}$ is quadratic moment of inertia of piezolayer to global axis $z$. Homogenized Youngs' moduli and homogenized density of beam with FGM core and piezoelectric layers are dependent only on axial location $x$.

\section{Piezoelectric beam finite element equations}

\subsection{Piezoelectric constitutive equations}

Piezoelectric constitutive equations describe the relation between mechanical and electrical quantities. The form of the constitutive equations depends on chosen mechanical and electrical quantities and can be expressed in two basic configurations. The first configuration is expressed by stress tensor components $\sigma_{k l}$ and vector components of electric intensity $E_{k}$ and has form

$$
\begin{gathered}
\varepsilon_{i j}=d_{i j k} E_{k}+s_{i j k l}^{E} \sigma_{k l} \\
D_{i}=\epsilon_{i k}^{\sigma} E_{k}+d_{i k l} \sigma_{k l}
\end{gathered}
$$

where $\varepsilon_{i j}$ are strain tensor components, $D_{i}$ are components of electric displacement vector, $d_{i j k}$ are tensor components of piezoelectric constants, $\epsilon_{i k}^{\sigma}$ are components of permittivity tensor on conditions constant mechanical stress and $s_{i j k l}^{E}$ are components of compliance tensor on conditions constant electric intensity [12].

The constitutive equations can be also expressed by strain tensor components $\varepsilon_{k l}$ and vector components of electric intensity $E_{k}$ and has form 


$$
\begin{gathered}
\sigma_{i j}=c_{i j k l}^{E} \varepsilon_{k l}-e_{i j k} E_{k} \\
D_{i}=e_{i k l} \varepsilon_{k l}+\epsilon_{i k}^{\varepsilon} E_{k}
\end{gathered}
$$

where new quantities are components of stiffness tensor $c_{i j k l}^{E}$ and components of piezoelectric modulus tensor $e_{i k l}$.

The other equations, which play important role, are relation between the components of strain tensor $\varepsilon_{i j}$ and components of deformation $v_{i}$ and relation between vector components of electric intensity $E_{i}$ and electric scalar potential $\phi$. These relations can be expressed as

$$
\begin{gathered}
\varepsilon_{i j}=1 / 2\left(v_{i, j}+v_{j, i}\right) \\
E_{i}=-\phi_{, i}
\end{gathered}
$$

Tensor equations (9) and (10) can be expressed in matrix forms [12], which is more suitable for finite element formulations, using symmetry of mechanical and electrical quantities as well as material properties. Matrix formulations of constitutive equations (10) can be expressed as

$$
\begin{gathered}
\boldsymbol{\sigma}=\boldsymbol{c}^{E} \boldsymbol{\varepsilon}-\boldsymbol{e}^{T} \boldsymbol{E} \\
\boldsymbol{D}=\boldsymbol{e} \boldsymbol{\varepsilon}+\boldsymbol{\epsilon}^{\varepsilon} \boldsymbol{E}
\end{gathered}
$$

where $\boldsymbol{\sigma}$ is vector (in mathematical meaning) of 6 stress tensor components, $\boldsymbol{D}$ is vector of 3 electric displacement vector components, $\boldsymbol{\varepsilon}$ is vector of 6 strain tensor components, $\boldsymbol{E}$ is vector of 3 electric intensity vector components, $\boldsymbol{c}^{E}$ is $6 \times 6$ matrix of mechanical properties, $\boldsymbol{\epsilon}^{\varepsilon}$ is $3 \times 3$ matrix of electrical properties and $\boldsymbol{e}$ is $3 \times 6$ matrix of piezoelectric properties.

\subsection{Piezoelectric FEM equations}

Piezoelectric governing equations for dynamic problems can by obtain by Hamilton's principle, which can be written in form

$$
\int_{t_{1}}^{t_{2}}(\delta L+\delta W) d t=0
$$

where $L$ is Lagrangian, $W$ is work of external mechanical and electrical forces and $t_{1}$ and $t_{2}$ defined considered time interval. Lagrangian of piezoelectric structure is given by equation

$$
L=T-U+W_{e}
$$

where $T, U$ and $W_{e}$ is kinetic energy, potential energy, and electric energy of investigated structure, respectively. They can be expressed as

$$
\begin{gathered}
T=\int_{(V)} \frac{1}{2} \rho \dot{\boldsymbol{v}}^{T} \dot{\boldsymbol{v}} d V \\
U=\int_{(V)} \frac{1}{2} \boldsymbol{\varepsilon}^{T} \boldsymbol{\sigma} d V \\
W_{e}=\int_{(V)} \frac{1}{2} \boldsymbol{E}^{T} \boldsymbol{D} d V
\end{gathered}
$$

where $\boldsymbol{v}$ is velocity vector with 3 components. Virtual work of external mechanical and electrical forces can be expressed as

$$
\delta W=\sum \delta \boldsymbol{v}^{T} \boldsymbol{F}-\sum \delta \phi Q
$$


where $\boldsymbol{v}$ is displacement vector with 3 components, $\boldsymbol{F}$ is force vector with 3 components, $\phi$ is electric scalar potential and $Q$ is electric charge.

Hamilton's principle (13) can be using equations (15), (16) and constitutive equation (12) expressed in following form

$$
\begin{gathered}
\int_{t_{1}}^{t_{2}}\left[-\int_{(V)} \rho \delta \boldsymbol{v}^{T} \ddot{\boldsymbol{v}} d V-\int_{(V)} \delta \boldsymbol{\varepsilon}^{T} \boldsymbol{c}^{E} \boldsymbol{\varepsilon} d V+\int_{(V)} \delta \boldsymbol{\varepsilon}^{T} \boldsymbol{e}^{T} \boldsymbol{E} d V+\int_{(V)} \delta \boldsymbol{E}^{T} \boldsymbol{e} \boldsymbol{\varepsilon} d V\right. \\
\left.+\int_{(V)} \delta \boldsymbol{E}^{T} \boldsymbol{\epsilon}^{\varepsilon} \boldsymbol{E} d V+\sum \delta \boldsymbol{v}^{T} \boldsymbol{F}-\sum \delta \phi Q\right] d t=0
\end{gathered}
$$

Relationship between displacement of point $\boldsymbol{v}$ and nodal displacement of finite element $\boldsymbol{v}^{e}$ and between electric scalar potential $\phi$ of point and nodal electric scalar potential of finite element $\boldsymbol{\phi}^{e}$ can be expressed by shape functions of element

$$
\begin{gathered}
\boldsymbol{v}=\boldsymbol{N}_{v} \boldsymbol{v}^{e} \\
\boldsymbol{\phi}=\boldsymbol{N}_{\phi} \boldsymbol{\phi}^{e}
\end{gathered}
$$

$\boldsymbol{N}_{v}$ and $\boldsymbol{N}_{\phi}$ are matrices with shape functions. Relationship between components of strain $\boldsymbol{\varepsilon}$ and components of nodal displacements $\boldsymbol{v}^{e}$ and relationship between components of electric intensity $\boldsymbol{E}$ and nodal electric scalar potential $\boldsymbol{\phi}^{e}$ have forms

$$
\begin{gathered}
\boldsymbol{\varepsilon}=\boldsymbol{B}_{v} \boldsymbol{v}^{e} \\
\boldsymbol{E}=-\boldsymbol{B}_{\phi} \boldsymbol{\phi}^{e}
\end{gathered}
$$

$\boldsymbol{B}_{v}$ and $\boldsymbol{B}_{\phi}$ are matrices with derivative of shape functions. Hamilton's principle (13) can be rewritten by equations (18) and (19) into form

$$
\begin{aligned}
\int_{t_{1}}^{t_{2}} \delta\left(\boldsymbol{v}^{e}\right)^{T}[- & \left(\int_{(V)} \boldsymbol{N}_{v}^{T} \rho \boldsymbol{N}_{v} d V\right) \ddot{\boldsymbol{v}}^{e}-\left(\int_{(V)} \boldsymbol{B}_{v}^{T} \boldsymbol{c}^{E} \boldsymbol{B}_{v} d V\right) \boldsymbol{v}^{e} \\
& \left.-\left(\int_{(V)} \boldsymbol{B}_{v}^{T} \boldsymbol{e}^{T} \boldsymbol{B}_{\phi} d V\right) \boldsymbol{\phi}^{e}++\sum \boldsymbol{N}_{v}^{T} \boldsymbol{F}\right] d t \\
& +\int_{t_{1}}^{t_{2}} \delta\left(\boldsymbol{\phi}^{e}\right)^{T}\left[-\left(\int_{(V)} \boldsymbol{B}_{\phi}^{T} \boldsymbol{e} \boldsymbol{B}_{v} d V\right) \boldsymbol{v}^{e}+\left(\int_{(V)} \boldsymbol{B}_{\phi}^{T} \boldsymbol{\epsilon}^{\varepsilon} \boldsymbol{B}_{\phi} d V\right) \boldsymbol{\phi}^{e}\right. \\
& \left.-\sum \boldsymbol{N}_{\phi}^{T} Q\right] d t=0
\end{aligned}
$$

FEM equations of individual element can be derived from (20) in form

$$
\left[\begin{array}{cc}
\boldsymbol{M}_{v v}^{e} & \mathbf{0}^{e} \\
\mathbf{0}^{e} & \mathbf{0}^{e}
\end{array}\right]\left[\begin{array}{l}
\ddot{\boldsymbol{v}}^{e} \\
\ddot{\boldsymbol{\phi}}^{e}
\end{array}\right]+\left[\begin{array}{ll}
\boldsymbol{K}_{v v}^{e} & \boldsymbol{K}_{v \phi}^{e} \\
\boldsymbol{K}_{\phi v}^{e} & \boldsymbol{K}_{\phi \phi}^{e}
\end{array}\right]\left[\begin{array}{l}
\boldsymbol{v}^{e} \\
\boldsymbol{\phi}^{e}
\end{array}\right]=\left[\begin{array}{l}
\boldsymbol{F}^{e} \\
\boldsymbol{Q}^{e}
\end{array}\right]
$$

The equation (21) represents dynamic behavior of piezoelectric material without mechanical damping. If the damping is considered, then the equation (21) has form

$$
\left[\begin{array}{cc}
\boldsymbol{M}_{v v}^{e} & \mathbf{0}^{e} \\
\mathbf{0}^{e} & \mathbf{0}^{e}
\end{array}\right]\left[\begin{array}{c}
\ddot{\boldsymbol{v}}^{e} \\
\ddot{\boldsymbol{\phi}}^{e}
\end{array}\right]+\left[\begin{array}{cc}
\boldsymbol{C}_{v v}^{e} & \mathbf{0}^{e} \\
\mathbf{0}^{e} & \mathbf{0}^{e}
\end{array}\right]\left[\begin{array}{c}
\dot{\boldsymbol{v}}^{e} \\
\dot{\boldsymbol{\phi}}^{e}
\end{array}\right]+\left[\begin{array}{ll}
\boldsymbol{K}_{v v}^{e} & \boldsymbol{K}_{v \phi}^{e} \\
\boldsymbol{K}_{\phi v}^{e} & \boldsymbol{K}_{\phi \phi}^{e}
\end{array}\right]\left[\begin{array}{l}
\boldsymbol{v}^{e} \\
\boldsymbol{\phi}^{e}
\end{array}\right]=\left[\begin{array}{c}
\boldsymbol{F}^{e} \\
\boldsymbol{Q}^{e}
\end{array}\right]
$$

where individual submatrices are defined as follows:

$$
\begin{aligned}
& \boldsymbol{M}_{v v}^{e}=\int_{(V)} \boldsymbol{N}_{v}^{T} \rho \boldsymbol{N}_{v} d V ; \boldsymbol{K}_{v v}^{e}=\int_{(V)} \boldsymbol{B}_{v}^{T} \boldsymbol{c}^{E} \boldsymbol{B}_{v} d V ; \boldsymbol{K}_{v \phi}^{e}=\int_{(V)} \boldsymbol{B}_{v}^{T} \boldsymbol{e}^{T} \boldsymbol{B}_{\phi} d V ; \\
& \boldsymbol{K}_{\phi v}^{e}=\int_{(V)} \boldsymbol{B}_{\phi}^{T} \boldsymbol{e} \boldsymbol{B}_{v} d V ; \boldsymbol{K}_{\phi \phi}^{e}=\int_{(V)} \boldsymbol{B}_{\phi}^{T} \boldsymbol{\epsilon}^{\varepsilon} \boldsymbol{B}_{\phi} d V ; \boldsymbol{C}_{v v}^{e}=\alpha \boldsymbol{M}_{v v}^{e}+\beta \boldsymbol{K}_{v v}^{e}
\end{aligned}
$$


$\boldsymbol{F}^{e}$ and $\boldsymbol{Q}^{e}$ represent nodal forces and nodal charges on considered element.

For whole investigated structure we can formally write

$$
\left[\begin{array}{cc}
\boldsymbol{M}_{v v} & \mathbf{0} \\
\mathbf{0} & \mathbf{0}
\end{array}\right]\left[\begin{array}{l}
\ddot{\boldsymbol{v}} \\
\ddot{\boldsymbol{\phi}}
\end{array}\right]+\left[\begin{array}{cc}
\boldsymbol{C}_{v v} & \mathbf{0} \\
\mathbf{0} & \mathbf{0}
\end{array}\right]\left[\begin{array}{l}
\dot{\boldsymbol{v}} \\
\dot{\boldsymbol{\phi}}
\end{array}\right]+\left[\begin{array}{ll}
\boldsymbol{K}_{v v} & \boldsymbol{K}_{v \phi} \\
\boldsymbol{K}_{\phi v} & \boldsymbol{K}_{\phi \phi}
\end{array}\right]\left[\begin{array}{l}
\boldsymbol{v} \\
\boldsymbol{\phi}
\end{array}\right]=\left[\begin{array}{l}
\boldsymbol{F} \\
\boldsymbol{Q}
\end{array}\right]
$$

\subsection{FEM equations of FGM beam with piezoelectric layers}

2D beam element with piezoelectric layers and FGM core with all degrees of freedom and all intermal mechanical and electrical forces is shown in Fig. 2.

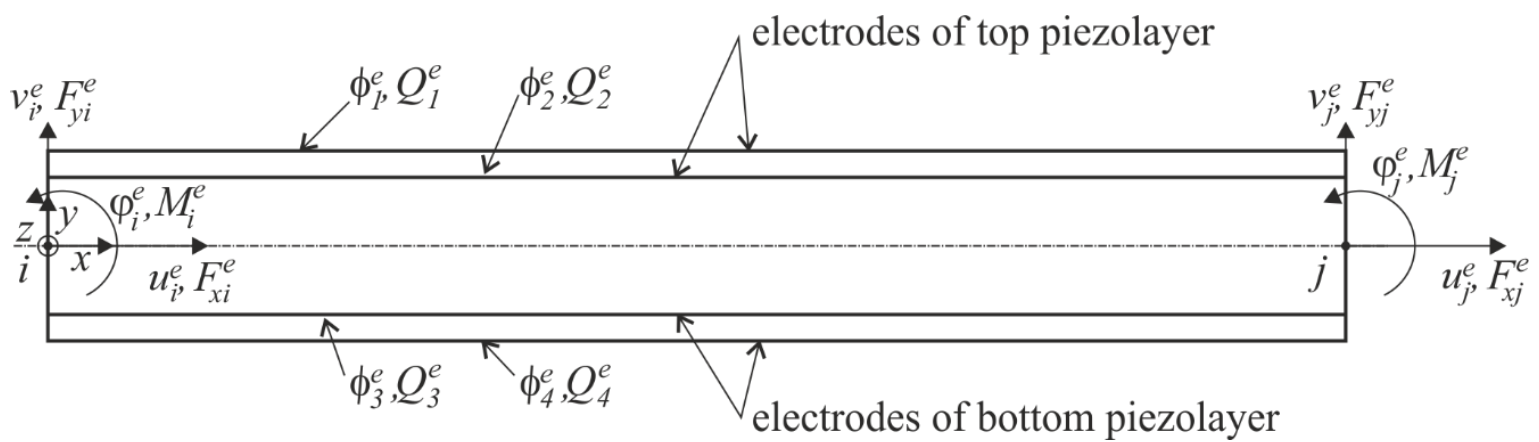

Fig. 2 2D beam element with piezoelectric layers - degrees of freedom and internal forces.

Length of beam element is $L^{e}$, cross-section of FGM core is $A_{F G M}$ and cross-section of piezolayer is $A_{p}$. Homogenized Youngs' moduli for axial loading and bending are computed according to equations (6) and (7) and homogenized density is computed according to equation (8). Mechanical degrees of freedom of element are displacements and rotations in both nodes $\left(u_{i}^{e}, v_{i}^{e}, \varphi_{i}^{e}, u_{j}^{e}, v_{j}^{e}, \varphi_{j}^{e}\right)$, electrical degree of freedom are electric potential on all 4 electrodes of both piezoelectric layers $\left(\phi_{1}^{e}, \phi_{2}^{e}, \phi_{3}^{e}, \phi_{4}^{e}\right)$. Internal element mechanical loads are forces and moments in both nodes $\left(F_{x i}^{e}, F_{y i}^{e}, M_{i}^{e}, F_{x j}^{e}, F_{y j}^{e}, M_{j}^{e}\right)$ and internal element electric loads are electric charge on all 4 electrodes of both piezoelectric layers $\left(Q_{1}^{e}, Q_{2}^{e}, Q_{3}^{e}, Q_{4}^{e}\right)[13]$.

FEM equation of 2D piezoelectric beam element with FGM core has formally the same equation as equation (22), but individual stiffness submatrices have different form, because they are using transfer constant and not classical shape functions. Mass submatrix $\boldsymbol{M}_{v v}^{e}$ can be constructed by classical shape functions [5].

The structural submatrix for the beam element with piezoelectric layers can be expressed in a form

$$
\boldsymbol{K}_{v v}^{e}=\left[\begin{array}{cccccc}
k_{u}^{\prime} & 0 & 0 & -k_{u}^{\prime} & 0 & 0 \\
0 & k_{v 2}^{\prime} & k_{v 3}^{\prime} & 0 & -k_{v 2}^{\prime} & k_{v 2} \\
0 & k_{v 3}^{\prime} & k_{v 33}^{\prime} & 0 & -k_{v 3}^{\prime} & k_{v 3} \\
-k_{u}^{\prime} & 0 & 0 & k_{u}^{\prime} & 0 & 0 \\
0 & -k_{v 2}^{\prime} & -k_{v 3}^{\prime} & 0 & k_{v 2}^{\prime} & -k_{v 2} \\
0 & k_{v 2} & k_{v 3} & 0 & -k_{v 2} & k_{v 23}
\end{array}\right]
$$

Computation of individual components of the structural submatrix contains the influence of FGM core and piezoelectric layers, where homogenized material properties of beam is considered. The calculation of individual components is identical for classical multilayer or FGM beam without piezoelectric layer and is described in [11]. The electrical submatrix for the beam element with piezoelectric layers can be expressed in a form [13] 


$$
\boldsymbol{K}_{\phi \phi}^{e}=\left[\begin{array}{cccc}
-\frac{A_{p} L \epsilon^{\varepsilon}}{h_{p}^{2}} & \frac{A_{p} L \epsilon^{\varepsilon}}{h_{p}^{2}} & 0 & 0 \\
\frac{A_{p} L \epsilon^{\varepsilon}}{h_{p}^{2}} & -\frac{A_{p} L \epsilon^{\varepsilon}}{h_{p}^{2}} & 0 & 0 \\
0 & 0 & -\frac{A_{p} L \epsilon^{\varepsilon}}{h_{p}^{2}} & \frac{A_{p} L \epsilon^{\varepsilon}}{h_{p}^{2}} \\
0 & 0 & \frac{A_{p} L \epsilon^{\varepsilon}}{h_{p}^{2}} & -\frac{A_{p} L \epsilon^{\varepsilon}}{h_{p}^{2}}
\end{array}\right]
$$

where $\epsilon^{\varepsilon}$ is permitivity of piezoelectric layer under constant strain.

Submatrices of piezoelectric coupling can be expressed in following forms [13]

$$
\boldsymbol{K}_{v \phi}^{e}=\left[\begin{array}{ccccc}
-\frac{A_{p} d_{21} E_{p}}{h_{p}} & \frac{A_{p} d_{21} E_{p}}{h_{p}} & -\frac{A_{p} d_{21} E_{p}}{h_{p}} & \frac{A_{p} d_{21} E_{p}}{h_{p}} \\
0 & 0 & 0 & 0 \\
\frac{A_{y} d_{21} E_{p}}{h_{p}} & -\frac{A_{y} d_{21} E_{p}}{h_{p}} & \frac{A_{y} d_{21} E_{p}}{h_{p}} & -\frac{A_{y} d_{21} E_{p}}{h_{p}} \\
\frac{A_{p} d_{21} E_{p}}{h_{p}} & -\frac{A_{p} d_{21} E_{p}}{h_{p}} & \frac{A_{p} d_{21} E_{p}}{h_{p}} & -\frac{A_{p} d_{21} E_{p}}{h_{p}} \\
-\frac{A_{y} d_{21} E_{p}}{h_{p}} & \frac{A_{y} d_{21} E_{p}}{h_{p}} & -\frac{A_{y} d_{21} E_{p}}{h_{p}} & \frac{A_{y} d_{21} E_{p}}{h_{p}}
\end{array}\right]
$$

where $A_{y}=\frac{1}{2} A_{p}\left(h_{F G M}+h_{p}\right), d_{21}$ and $e_{21}$ are piezoelectric constants.

\subsection{Sensor and actuator FEM equations}

The matrix equation (23) of investigated structure can be rewritten in the form

$$
\begin{gathered}
\boldsymbol{M}_{v v} \ddot{\boldsymbol{v}}+\boldsymbol{C}_{v v} \dot{\boldsymbol{v}}+\boldsymbol{K}_{v v} \boldsymbol{v}+\boldsymbol{K}_{v \phi}(\boldsymbol{\phi})_{\text {sens }}=\boldsymbol{F}-\boldsymbol{K}_{v \phi}(\boldsymbol{\phi})_{\mathrm{actu}} \\
\boldsymbol{K}_{\phi v} \boldsymbol{v}+\boldsymbol{K}_{\phi \phi}(\boldsymbol{\phi})_{\text {sens }}=(\boldsymbol{Q})_{\text {sens }} \\
\boldsymbol{K}_{\phi v} \boldsymbol{v}+\boldsymbol{K}_{\phi \phi}(\boldsymbol{\phi})_{\mathrm{actu}}=(\boldsymbol{Q})_{\mathrm{actu}}
\end{gathered}
$$

where $(\boldsymbol{\phi})_{\text {sens }}$ and $(\boldsymbol{Q})_{\text {sens }}$ is electric potential and electric charge of sensors, respectively, and $(\boldsymbol{\phi})_{\text {actu }}$ and $(\boldsymbol{Q})_{\text {actu }}$ is electric potential and electric charge of actuators, respectively. If electrodes on piezoelectric sensors are short circuit, i.e., $(\boldsymbol{\phi})_{\text {sens }}=\mathbf{0}$, and electrodes on piezoelectric actuators are open circuit, i.e., $(\boldsymbol{Q})_{\text {actu }}=\mathbf{0}$, then equations of investigated structure have form 


$$
\begin{gathered}
\boldsymbol{M}_{v v} \ddot{\boldsymbol{v}}+\boldsymbol{C}_{v v} \dot{\boldsymbol{v}}+\boldsymbol{K}_{v v} \boldsymbol{v}=\boldsymbol{F}-\boldsymbol{K}_{v \phi}(\boldsymbol{\phi})_{a c t u} \\
\boldsymbol{K}_{\phi v} \boldsymbol{v}=(\boldsymbol{Q})_{\text {sens }}
\end{gathered}
$$

Equation (31) is used to calculate the deformation of the whole structure, which is loaded by external forces on the structure as well as the electric potential on the piezoelectric actuator. Equation (32) is used to calculate the electric charge, which is collected on the piezoelectric sensor. If electrodes on piezoelectric sensors and actuators are open circuit, i.e., $(\boldsymbol{Q})_{\text {sens }}=\mathbf{0}$ and $(\boldsymbol{Q})_{\text {actu }}=\mathbf{0}$, then these equations have form

$$
\begin{aligned}
\boldsymbol{M}_{v v} \ddot{\boldsymbol{v}}+\boldsymbol{C}_{v v} \dot{\boldsymbol{v}}+\boldsymbol{K}_{v v} \boldsymbol{v}+\boldsymbol{K}_{v \phi}(\boldsymbol{\phi})_{\text {sens }} & =\boldsymbol{F}-\boldsymbol{K}_{v \phi}(\boldsymbol{\phi})_{a c t u} \\
\boldsymbol{K}_{\phi v} \boldsymbol{v}+\boldsymbol{K}_{\phi \phi}(\boldsymbol{\phi})_{\text {sens }} & =\mathbf{0}
\end{aligned}
$$

Equations (33) and (34) can be rewritten to form

$$
\begin{gathered}
\boldsymbol{M}_{v v} \ddot{\boldsymbol{v}}+\boldsymbol{C}_{v v} \dot{\boldsymbol{v}}+\left(\boldsymbol{K}_{v v}-\boldsymbol{K}_{v \phi} \boldsymbol{K}_{\phi \phi}^{-1} \boldsymbol{K}_{\phi v}\right) \boldsymbol{v}=\boldsymbol{F}-\boldsymbol{K}_{v \phi}(\boldsymbol{\phi})_{\mathrm{actu}} \\
\boldsymbol{K}_{\phi v} \boldsymbol{v}+\boldsymbol{K}_{\phi \phi}(\boldsymbol{\phi})_{\text {sens }}=\mathbf{0}
\end{gathered}
$$

Equation (35) is used to calculate the deformation of investigated structure, which is loaded by external forces on the structure as well as the electric potential on the piezoelectric actuator. Equation (36) is used to calculate the electric potential that is induced on the piezoelectric sensor. Developed FEM equations were implemented in FEM code MultiFEM, which is programmed in software Mathematica [10].

\section{$4 \quad$ Reduced state-space model}

Smart structures made from piezoelectric material, are usually connected to controller in order to have required behavior of structure. For this purpose, FEM equations are transformed to state-space equations [14]. The displacement of the nodal points can be expressed as

$$
\boldsymbol{v}=\boldsymbol{Z w}
$$

where the matrix $\boldsymbol{Z}$ contains eigenshapes and $\boldsymbol{w}$ is vector of amplitudes of these eigenshapes. Using the transformation equation (37), equations (31) and (32) of investigated structure, where electrodes on piezoelectric sensors are short circuit and electrodes on piezoelectric actuators are open circuit, can be rewritten in form

$$
\begin{gathered}
\boldsymbol{M}_{v v} Z \ddot{\boldsymbol{w}}+\boldsymbol{C}_{v v} \boldsymbol{Z} \dot{\boldsymbol{w}}+\boldsymbol{K}_{v v} Z \boldsymbol{w}=\boldsymbol{F}-\boldsymbol{K}_{v \phi}(\boldsymbol{\phi})_{\mathrm{actu}} \\
(\boldsymbol{Q})_{\mathrm{sens}}=\boldsymbol{K}_{\phi v} \boldsymbol{Z} \boldsymbol{w}
\end{gathered}
$$

Using orthogonality property of mode shapes

$$
\begin{gathered}
\boldsymbol{Z}^{T} \boldsymbol{M}_{v v} \boldsymbol{Z}=\operatorname{diag}\left(\mu_{k}\right) \\
\boldsymbol{Z}^{T} \boldsymbol{K}_{v v} \boldsymbol{Z}=\operatorname{diag}\left(\mu_{k} \omega_{k}^{2}\right) \\
\boldsymbol{Z}^{T} \boldsymbol{C}_{v v} \boldsymbol{Z}=\operatorname{diag}\left(2 \xi_{k} \mu_{k} \omega_{k}\right)
\end{gathered}
$$

equations (38) and (39) can be rewritten in form

$$
\begin{gathered}
\boldsymbol{\mu} \ddot{\boldsymbol{w}}+2 \xi \boldsymbol{\mu} \boldsymbol{\omega} \dot{\boldsymbol{w}}+\boldsymbol{\mu} \boldsymbol{\omega}^{2} \boldsymbol{w}=\boldsymbol{Z}^{T} \boldsymbol{F}-\boldsymbol{Z}^{T} \boldsymbol{K}_{v \phi}(\boldsymbol{\phi})_{\mathrm{actu}} \\
(\boldsymbol{Q})_{\mathrm{sens}}=\boldsymbol{K}_{\phi v} \boldsymbol{Z} \boldsymbol{w}
\end{gathered}
$$

where $\boldsymbol{\mu}, \boldsymbol{\omega}$ and $\boldsymbol{\xi}$ represent matrix of modal masses, matrix of modal frequencies and matrix of modal damping ratios of structure, respectively. Equations (43) and (44) can be rewritten into form

$$
\left[\begin{array}{c}
\dot{\boldsymbol{W}} \\
\ddot{\boldsymbol{W}} \\
(\boldsymbol{Q})_{\text {sens }}
\end{array}\right]=\left[\begin{array}{cc}
\mathbf{0} & \boldsymbol{I} \\
-\boldsymbol{\omega}^{2} & -2 \xi \boldsymbol{\omega} \\
\boldsymbol{K}_{\phi v} \boldsymbol{Z} & \mathbf{0}
\end{array}\right]\left[\begin{array}{l}
\boldsymbol{W} \\
\dot{\boldsymbol{w}}
\end{array}\right]+\left[\begin{array}{cc}
\mathbf{0} & \mathbf{0} \\
-\boldsymbol{\mu}^{-1} \boldsymbol{Z}^{T} \boldsymbol{K}_{v \phi} & \boldsymbol{\mu}^{-1} \boldsymbol{Z}^{T} \\
\mathbf{0} & \mathbf{0}
\end{array}\right]\left[\begin{array}{c}
(\boldsymbol{\phi})_{\mathrm{actu}} \\
\boldsymbol{F}
\end{array}\right]
$$


Equation (45) can be formally written as state-space model

$$
\begin{aligned}
& \dot{x}=A x+B u \\
& y=C x+D u
\end{aligned}
$$

where individual matrices $\boldsymbol{A}, \boldsymbol{B}, \boldsymbol{C}$ and $\boldsymbol{D}$ have form

$$
\begin{aligned}
& A=\left[\begin{array}{cc}
0 & I \\
-\omega^{2} & -2 \xi \omega
\end{array}\right] \quad B=\left[\begin{array}{cc}
0 & 0 \\
-\mu^{-1} Z^{T} K_{v \phi} & \mu^{-1} Z^{T}
\end{array}\right] \\
& C=\left[\begin{array}{ll}
K_{\phi v} Z & 0
\end{array}\right] \quad D=\left[\begin{array}{ll}
0 & 0
\end{array}\right]
\end{aligned}
$$

and vector $\boldsymbol{x}=\left[\begin{array}{cc}\boldsymbol{w} & \dot{\boldsymbol{w}}\end{array}\right]^{T}$ is state vector, $\boldsymbol{y}=(\boldsymbol{Q})_{\text {sens }}$ is output vector and $\boldsymbol{u}=$

$\left[\begin{array}{ll}\boldsymbol{w}(\boldsymbol{\phi})_{\text {actu }} & \boldsymbol{F}\end{array}\right]^{T}$ is input vector. If electrodes on piezoelectric sensors and actuators are open circuit, matrices $\boldsymbol{A}$ and $\boldsymbol{C}$ have shape

$$
A=\left[\begin{array}{cc}
0 & I \\
-\omega^{2}+Z^{T} K_{v \phi} K_{\phi \phi}^{-1} K_{\phi v} Z & -2 \xi \omega
\end{array}\right] \quad C=\left[\begin{array}{ll}
-K_{\phi \phi}^{-1} K_{\phi v} Z & 0
\end{array}\right]
$$

and output vector is $\boldsymbol{y}=(\boldsymbol{\phi})_{\text {sens }}$.

When in the transformation process from the FEM model to the state-space model only selected eigenmodes and appropriate eigenfrequencies of system are considered, we obtain reduced modal truncation model [6].

\section{$5 \quad$ Control of MOR model}

Input vector $\boldsymbol{u}$ can be split into external input $\boldsymbol{u}_{e}$ and controller input $\boldsymbol{u}_{c}$. The control for a linear system with full-state feedback [15], which is shown in Fig. 3, is given by proportional control law

$$
\boldsymbol{u}_{\boldsymbol{c}}=-\boldsymbol{K} \boldsymbol{x}
$$

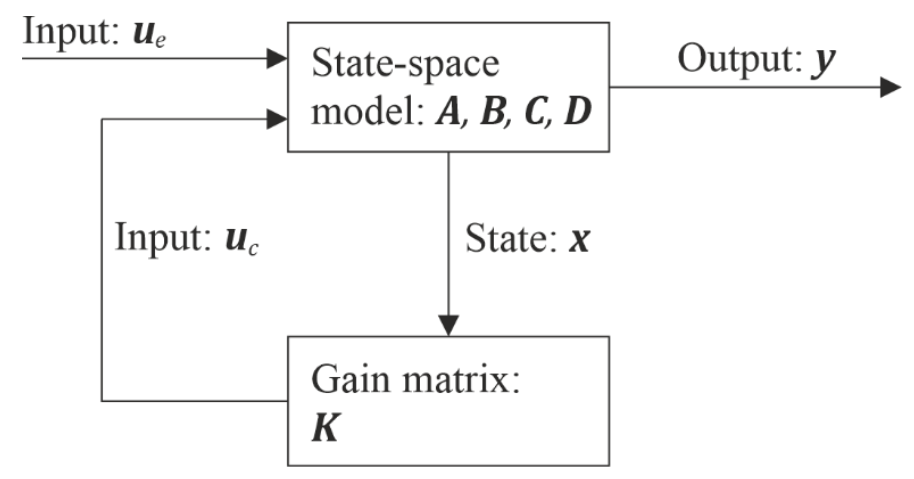

Fig. 3 Control of system with full-state feedback.

The goal of the linear quadratic control law is to design the gain matrix $\boldsymbol{K}$ so that the state of the system $\boldsymbol{x}$ converges to the zero state as quickly as possible, but with the least possible effort. Mathematically, this can be expressed using the so-called cost function in the form

$$
J=\frac{1}{2} \int_{0}^{\infty}\left(x^{T} Q x+u_{c}^{T} R u_{c}\right) d t
$$

and the goal is to construct $\boldsymbol{u}_{c}=-\boldsymbol{K} \boldsymbol{x}$ in such a way to minimize $J=\lim _{t \rightarrow \infty} J(t)$. The resulting full-state feedback controller is called a linear quadratic regulator (LQR), since it is a linear control law that minimizes a quadratic cost function to regulate the system. 
Matrix $\boldsymbol{Q}$ is positive semidefinite matrix and $\boldsymbol{R}$ is positive matrix and they represent weights of state and input vectors in the process of minimization of a cost function $J$. These matrices are often diagonal, and the diagonal elements may be tuned to change the relative importance of the control objectives.

\section{$6 \quad$ Numerical examples}

Numerical experiments contain two examples, both of which contain a functionally graded beam on which piezoelectric layers are placed. The material composition of the functionally graded beam as well as the piezoelectric layers are the same in both investigated examples. Since the mathematical models use the homogenization of the material properties of both the FGM beam without piezoelectric layers and the FGM beam with piezoelectric layers, it is necessary to create these homogenized material properties - this is discussed in section 6.1. The created homogenized material properties are then used in the analysis of a simple FGM beam with piezoelectric layers - section 6.2 as well as in the analysis of FGM beam structure with piezoelectric layers - section 6.3.

\subsection{Homogenized material properties}

Beam, shown in Fig. 1, consists of beam core and outer piezoelectric layers. The height of the core is $h_{F G M}=0.01 \mathrm{~m}$ and the height of the piezoelectric layer is $h_{p}=0.001 \mathrm{~m}$. The width of the analysed beam is $b=0.01 \mathrm{~m}$ and length is $0.1 \mathrm{~m}$.

Beam core is made of functionally graded materials. Material of matrix (index $m$ ) is $\mathrm{NiFe}$ with constant density and Young's modulus and material of fibre (index $f$ ) is tungsten with constant density and Young's modulus:

- Young's modulus: $E_{m}=255 \mathrm{GPa}, E_{f}=400 \mathrm{GPa}$

- density: $\rho_{m}=9200 \mathrm{~kg} / \mathrm{m}^{3}, \rho_{f}=19300 \mathrm{~kg} / \mathrm{m}^{3}$

Volume fractions of both constituents $v_{m}(x, y)$ and $v_{f}(x, y)$ vary along the length and height of beams ( $x$ is longitudinal axis and $y$ is transversal axis of beam - see Fig. 1) according to equations:

$$
\begin{gathered}
v_{m}(x, y)=-1 . \overline{3} \times 10^{8} x^{3} y^{2}+1333 . \overline{3} x^{3}+2 \times 10^{7} x^{2} y^{2}-200 x^{2}-40000 y^{2}+1[-] \\
v_{f}(x, y)=1 . \overline{3} \times 10^{8} x^{3} y^{2}-1333 . \overline{3} x^{3}-2 \times 10^{7} x^{2} y^{2}+200 x^{2}+40000 y^{2}[-]
\end{gathered}
$$

Both functions of volume fractions for beam core with length $0.1 \mathrm{~m}$ and height $0.01 \mathrm{~m}$ are shown in Fig. 4.
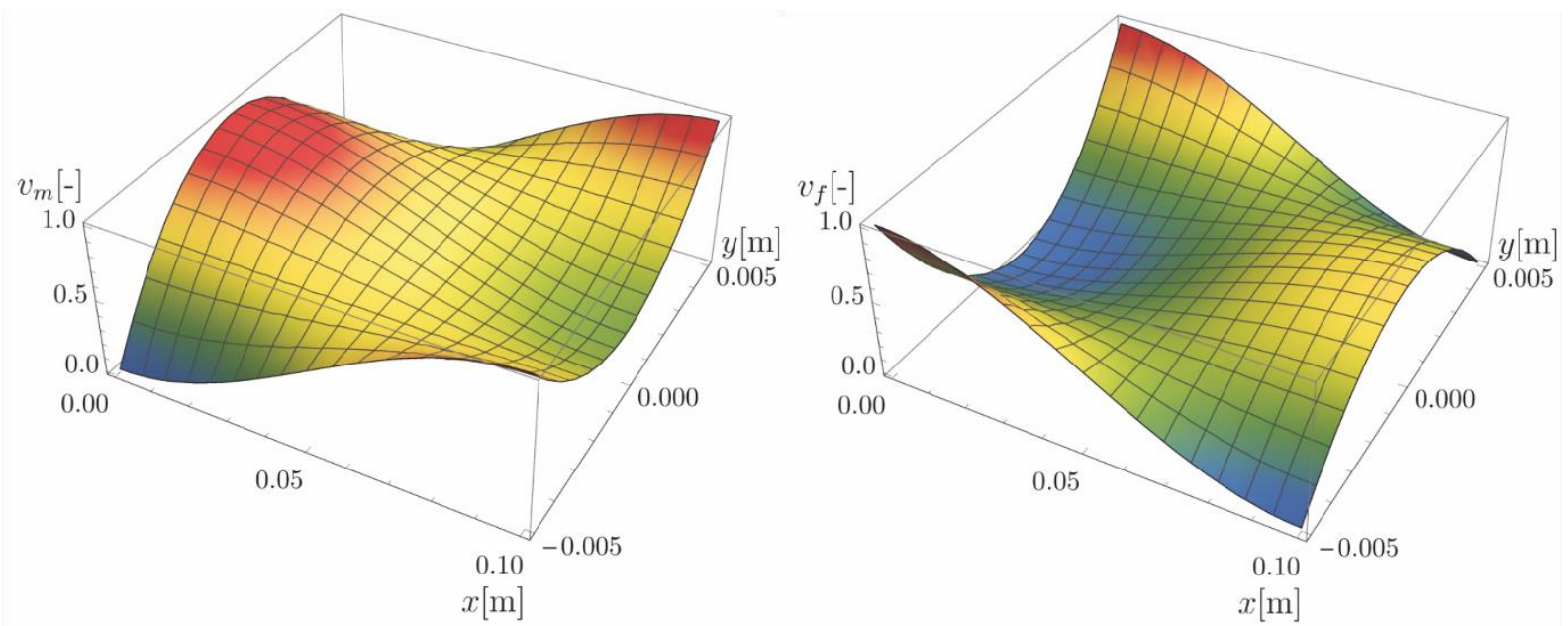

Fig. 4 Volume fraction of matrix - left, volume fraction of fibre - right. 
Effective material properties of FGM are defined by material properties of constituents and their variations and Young's modulus and density of considered FGM (equations (1) and (2)) have form

$$
\begin{gathered}
E_{F G M}(x, y)=1.9 \overline{3} \times 10^{19} x^{3} y^{2}-1.9 \overline{3} \times 10^{14} x^{3}-2.9 \times 10^{18} x^{2} y^{2}+2.9 \times 10^{13} x^{2} \\
+5.8 \times 10^{15} y^{2}+2.55 \times 10^{11}[\mathrm{~Pa}] \\
\rho_{F G M}(x, y)=1.34667 \times 10^{12} x^{3} y^{2}-1.34667 \times 10^{7} x^{3}-2.02 \times 10^{11} x^{2} y^{2} \\
+2.02 \times 10^{6} x^{2}+4.04 \times 10^{8} y^{2}+9200\left[\mathrm{~kg} / \mathrm{m}^{3}\right]
\end{gathered}
$$

Homogenized material properties of investigated FGM beams can be calculated by defined cross-section parameters of beams and by effective material properties (equations (3), (4) and (5)) and have following forms:

$$
\begin{gathered}
E_{F G M}^{N}(x)=-3 . \overline{2} \times 10^{13} x^{3}+4.8 \overline{3} \times 10^{12} x^{2}+3.0 \overline{3} \times 10^{11}[\mathrm{~Pa}] \\
E_{F G M}^{M}(x)=9 . \overline{6} \times 10^{13} x^{3}-1.45 \times 10^{13} x^{2}+3.42 \times 10^{11}[\mathrm{~Pa}] \\
\rho_{F G M}(x)=-2.2 \overline{4} \times 10^{6} x^{3}+33666 . \overline{6} x^{2}+12566 . \overline{6}\left[\mathrm{~kg} / \mathrm{m}^{3}\right]
\end{gathered}
$$

$E_{F G M}^{N}(x)$ and $E_{F G M}^{M}(x)$ represent homogenized Young's modulus for axial loading and for bending and $\rho_{F G M}(x)$ is homogenized density of FGM core, respectively.

Piezoelectric layers in investigated beams are made from PZT5A piezoelectric material. PZT5A is orthotropic material and has following material properties (direction of poling has index 3):

- mechanical properties:

○ Young's moduli: $E_{1}=61 \mathrm{GPa}, E_{2}=61 \mathrm{GPa}, E_{3}=53.2 \mathrm{GPa}$

○ Poisson numbers: $\mu_{12}=0.35, \mu_{13}=0.38, \mu_{23}=0.38$

○ shear moduli: $G_{12}=22.6 \mathrm{GPa}, G_{13}=21.1 \mathrm{GPa}, G_{23}=21.1 \mathrm{GPa}$

○ density: $7750 \mathrm{~kg} / \mathrm{m}^{3}$

- piezoelectric properties: $d_{31}=-171 \times 10^{-12} \mathrm{C} / \mathrm{N}, d_{33}=374 \times 10^{-12} \mathrm{C} / \mathrm{N}$, $d_{15}=584 \times 10^{-12} \mathrm{C} / \mathrm{N}, d_{24}=584 \times 10^{-12} \mathrm{C} / \mathrm{N}$

- relative permittivity: $\epsilon_{11}^{\sigma}=1728.8, \epsilon_{22}^{\sigma}=1728.8, \epsilon_{33}^{\sigma}=1694.9$

Homogenized Young's modulus for axial loading $E^{H N}(x)$ and bending $E^{H N}(x)$ and density $\rho(x)$ of beam with FGM core and piezoelectric layers can be calculated by (6), (7) and (8) and they have form

$$
\begin{gathered}
E^{N}(x)=-2.685 \times 10^{13} x^{3}+4.027 \times 10^{12} x^{2}+2.629 \times 10^{11}[\mathrm{~Pa}] \\
E^{M}(x)=5.594 \times 10^{13} x^{3}-8.391 \times 10^{12} x^{2}+2.236 \times 10^{11}[\mathrm{~Pa}] \\
\rho(x)=-1.871 \times 10^{6} x^{3}+305833 . \overline{3} x^{2}+11762.1 \overline{6}\left[\mathrm{~kg} / \mathrm{m}^{3}\right]
\end{gathered}
$$

\subsection{Simple FGM beam with piezoelectric layers}

\section{Analysed system}

Fig. 5 left shows the analysed FGM beam structure on which the piezoelectric actuators and sensors are placed. The length of FGM beam is 0.1, the length of sensor and actuator is $0.01 \mathrm{~m}$ and they are placed $0.01 \mathrm{~m}$ and $0.03 \mathrm{~m}$ from fixed left end, respectively. The discretized FEM model is shown in Fig. 5 right. 


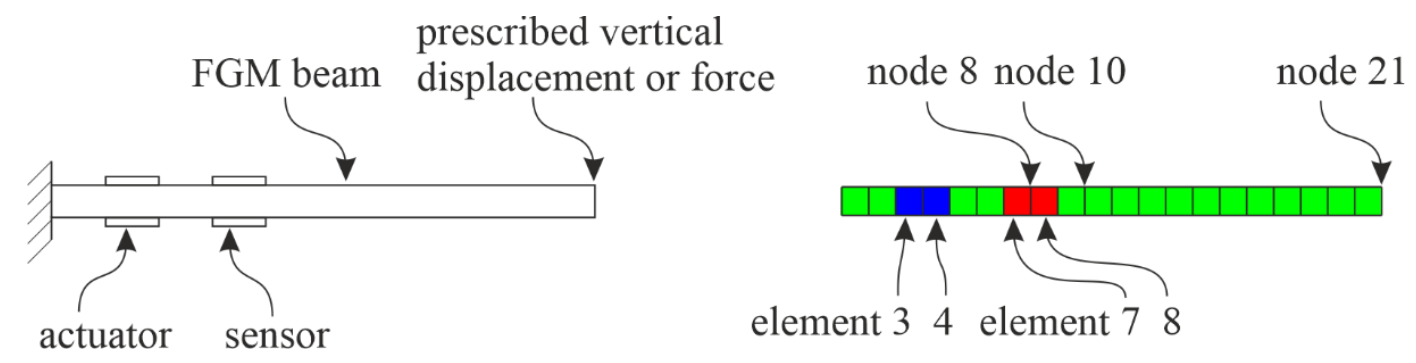

Fig. 5 Analysed FGM beam system with piezoelectric actuators and sensors - left, discretized FEM model - right.

Homogenized material properties of FGM core without piezoelectric layers as well as FGM core with piezoelectric layers were defined in section 6.1. Damping of structure is considered as Rayleigh damping with mass and stiffness constants $3 \times 10^{-5} \mathrm{~s}^{-1}$ and $3 \times 10^{-5}$ $\mathrm{s}$, respectively. The inner electrodes of piezoelectric layers are grounded.

The goal of the analysis is to perform a static and dynamic FEM analysis of the beam structure with different excitations and then create a MOR model and compare the results obtained from the MOR model and the FEM model. The analysis also includes a design of LQR controller of the structure to minimize the vibration of the structure.

\section{Static FEM analysis}

Static FEM analysis is considered as an initial analysis that defines the initial state for the selected transient analysis. The deformed state is defined by the prescribed vertical displacement at node 8 with a value $-0.25 \times 10^{-5} \mathrm{~m}$ and at node 21 with a value of $1 \times 10^{-5}$ $\mathrm{m}-$ see Fig. 5. Calculated deformed shape is shown in Fig. 6.

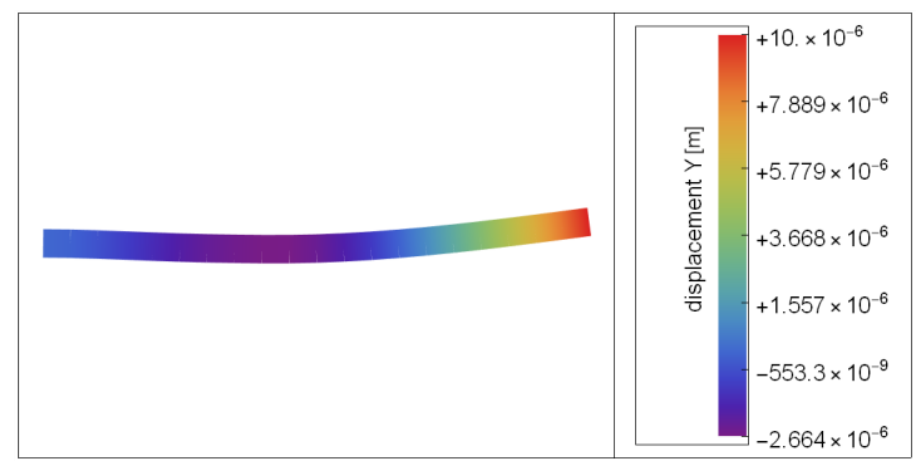

Fig. 6 Static deformed shape of analysed beam structure.

\section{Modal FEM analysis}

The goal of the modal analysis is to obtain the first four bending eigenfrequencies and the corresponding eigenmodes of the analysed beam structure. These eigenfrequencies and eigenmodes are subsequently used both in the creation of a reduced model of the system and in the definition of the modal initial state of the system. The first four eigenshapes are shown in Fig. 7. First four bending eigenfrequencies of analysed systems are $188.4 \mathrm{~Hz}, 988.5 \mathrm{~Hz}$, $5532 \mathrm{~Hz}$ and $9765 \mathrm{~Hz}$. 


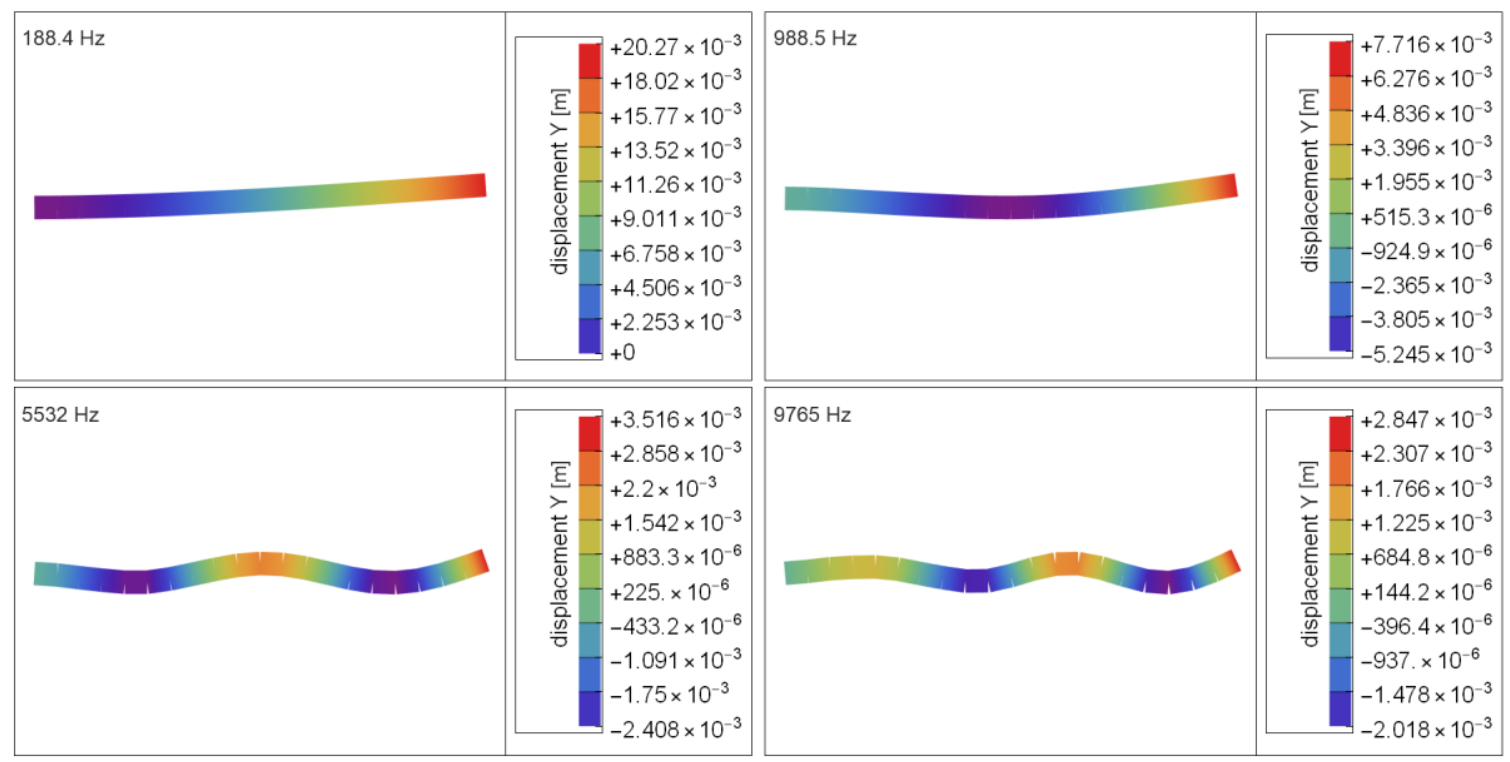

Fig. 7 The first four bending eigenmodes.

\section{Harmonic FEM analysis}

The response of the analysed structure to the harmonic vertical force with amplitude $20 \mathrm{~N}$ acting at the free end of the structure - node 21 (see Fig. 5) is shown in Fig. 8.
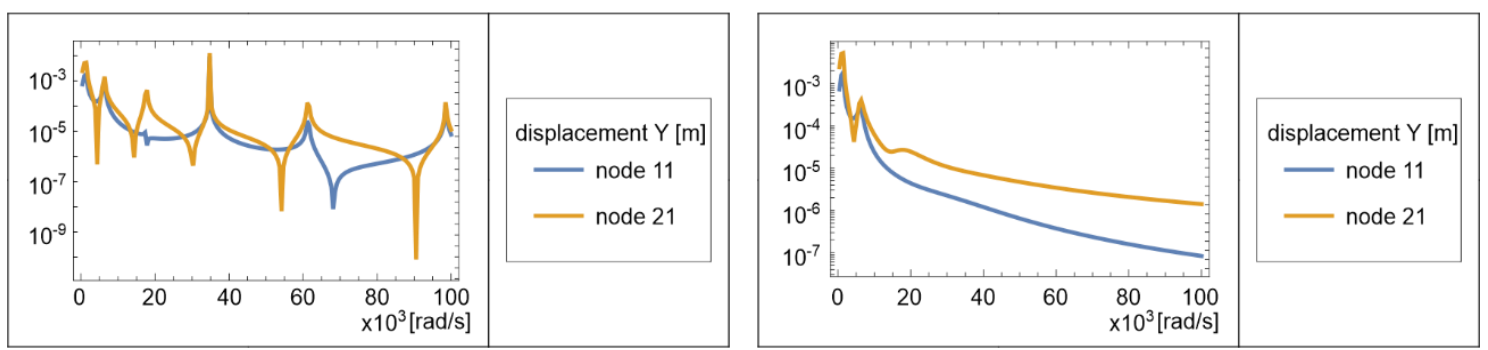

Fig. 8 Response of system to harmonic vertical force, without considering damping - left, with damping - right.

As can be seen from the harmonic analysis of piezoelectric system with damping, the dominant natural frequencies are the first four. For this reason, the first four bending eigenshapes and eigenfrequencies of the analysed system are used in the reduced model.

\section{Transient analysis}

In the transient analysis, 3 different excitations were considered: excitation by initial conditions, excitation by external force, excitation by electric potential on actuators. Investigated time for transient analysis is $0.025 \mathrm{~s}$.

Excitation by initial conditions: the initial conditions of the system are defined by the static deformation, which was presented at the subsection Static analysis. Newmark's numerical method was used to calculate the system response. The displacements of the nodes 10 and 21 and the electric charge on the electrodes on the elements 7 and 8 are shown in Fig. 9. 

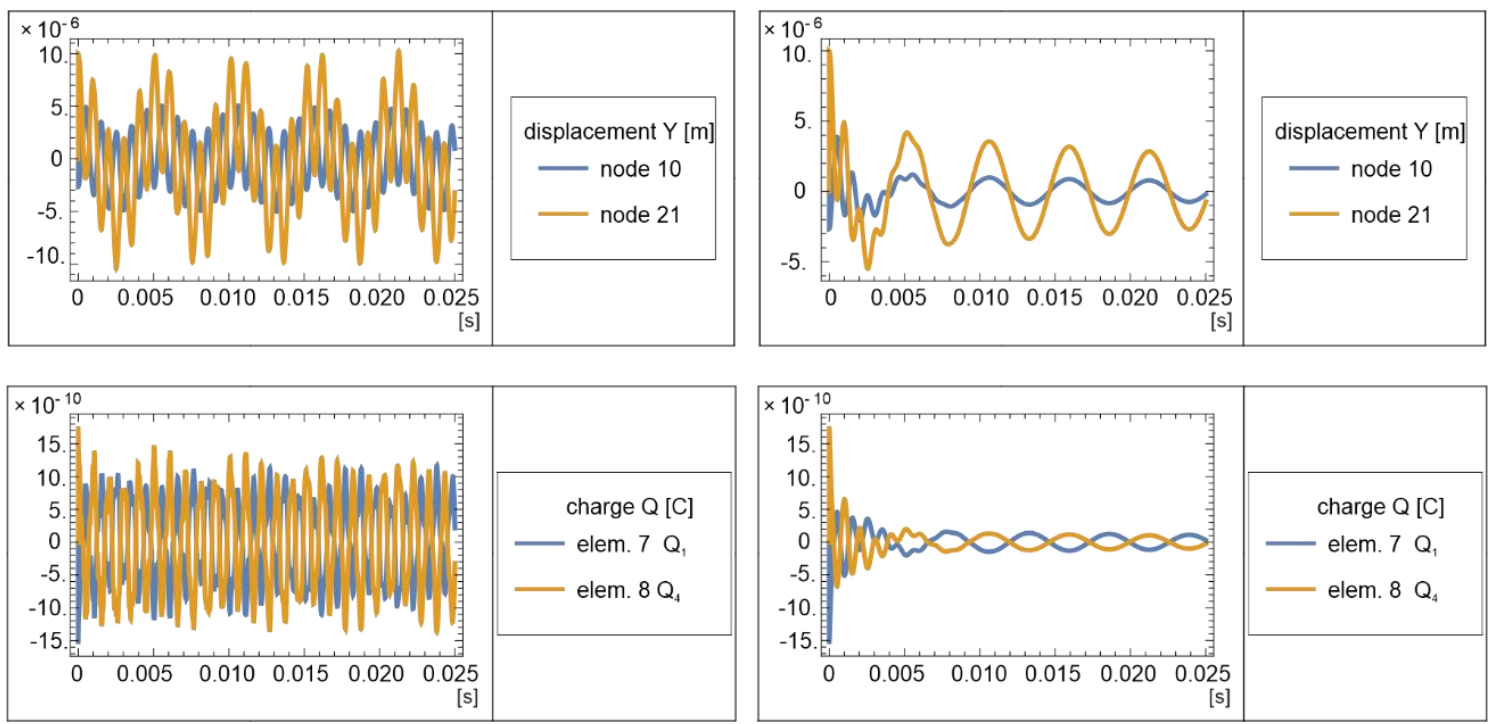

Fig. 9: Excitation by initial conditions - displacements and the electric charge, without considering damping - left, with damping - right.

Excitation by external force: the external force is located at the free end of the system node 21 (see Fig. 5). The system had zero deformation and zero velocity at the beginning of investigated time. The force has a harmonic character with frequency $80 \mathrm{~Hz}$ and an amplitude of $10 \mathrm{~N}$. The displacement of nodes 10 and 21 as well as the electric charge on elements 7 and 8 of damped system are shown in Fig. 10.
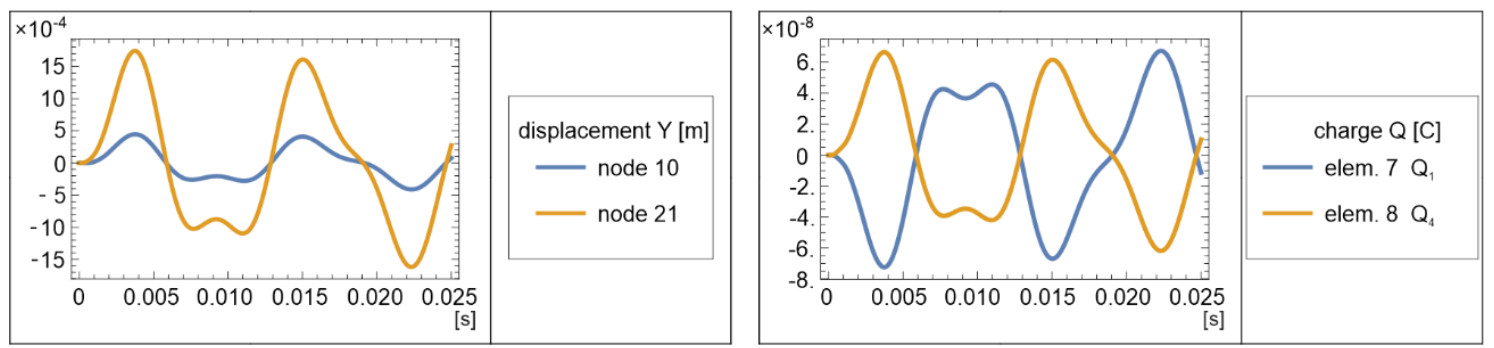

Fig. 10 Excitation by external force - displacements and the electric charge.

Excitation by electric potential on actuators: piezoelectric actuators are located closer to the left fixed end - elements 3 and 4 - see Fig. 5. The system had zero deformation and zero velocity at the beginning of investigated time. The inner electrodes are grounded, an electric harmonic potential with an amplitude of $100 \mathrm{~V}$ and a frequency of $40 \mathrm{~Hz}$ is prescribed at the outer electrodes. The displacement of the nodes 10 and 21 as well as the electric charge on the elements 7 and 8 of damped system are shown in Fig. 11.
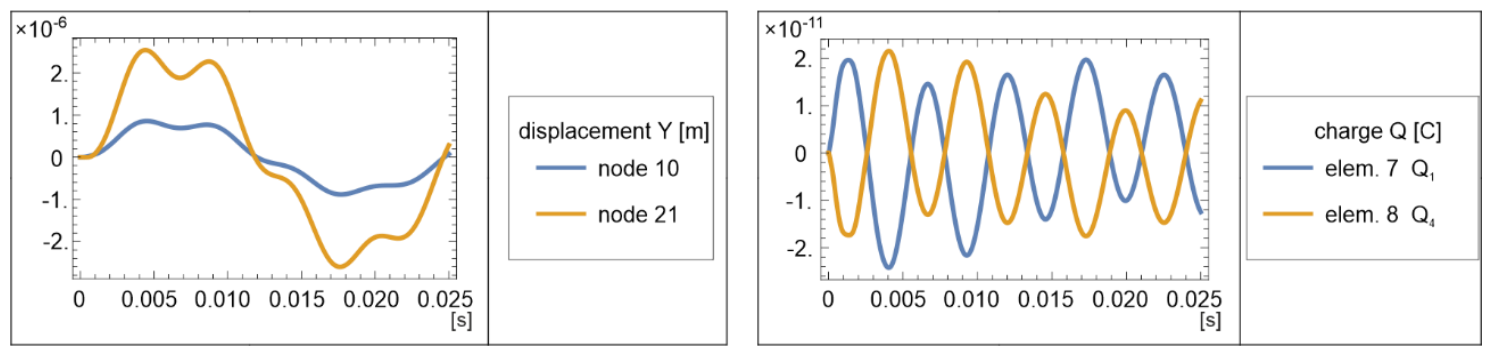

Fig. 11 Excitation by electric potential on actuators - displacements and the electric charge.

\section{MOR state-space model}

Based on the modal analysis results, a reduced state-space model described by equation (45) was created using the first four bending eigenfrequencies and their corresponding 
eigenshapes. In the model, the damping of system was defined as in the FEM model. In the dynamic analysis of the reduced state-space model, all excitations which were used in the transient FEM analysis with the same set-up, were considered. Initial deformations, external vertical force located at the free end of the beam structure and electric potential on actuators had to be transformed into modal space. Fig. 12 shows the vertical displacement of the node 21 caused by initial deformation of system, which was calculated from the full FEM model blue line and from the reduced state-space model - brown rings, considering a system without damping as well as a system with damping.
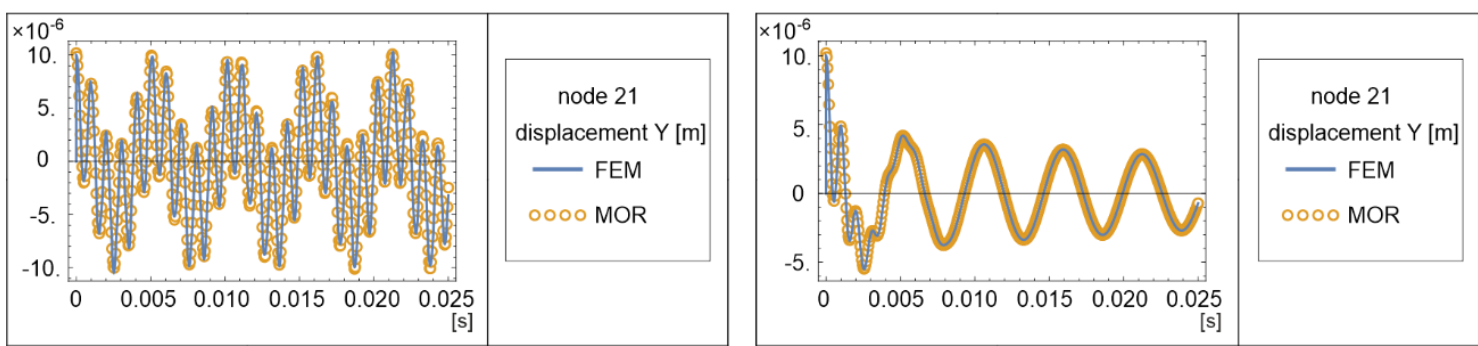

Fig. 12 Vertical displacement of the node 21 calculated by FEM model - blue line and by MOR model - brown rings, without considering damping - left, with damping - right.

Fig. 13 shows time variation of the vertical displacement of the node 21 in the model with the damping under the load of the vertical harmonic force - the left figure, and under the load of the electric harmonic potential on the piezoelectric actuator - the right figure.
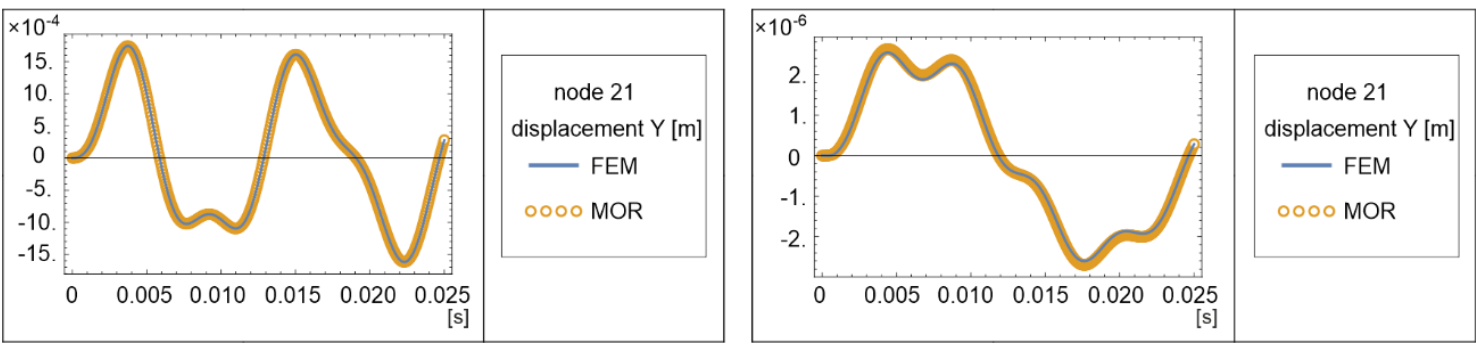

Fig. 13 Vertical displacement of the node 21 calculated by FEM model - blue line and by MOR model - brown rings, system loaded by vertical harmonic force - left, system loaded by electric harmonic potential on the piezoelectric actuator - right.

The blue line in Fig. 13 represents the results from the FEM analysis and the brown rings represent the results from the reduced state-space model. As can be seen from Fig. 12 and 13, MOR state-space model very faithfully describes the behaviour of the system and the statespace model have significantly fewer degrees of freedom than the full FEM model.

\section{Control law}

A reduced state-space model was used to design the LQR controller, with a state weight of 50,000 and an input weight of 1 . Based on the parameters thus selected, the gain matrix $\boldsymbol{K}$ was calculated and then a feedback state-space model was built. Subsequently, the state-space model with feedback was analysed, considering the initial conditions defined by static FEM analysis and transformed into modal space. Comparison of the system response, i.e., the vertical deflection of the node 21 for the system without control - blue line and for the system with control - brown line, is shown in Fig. 14 left. 

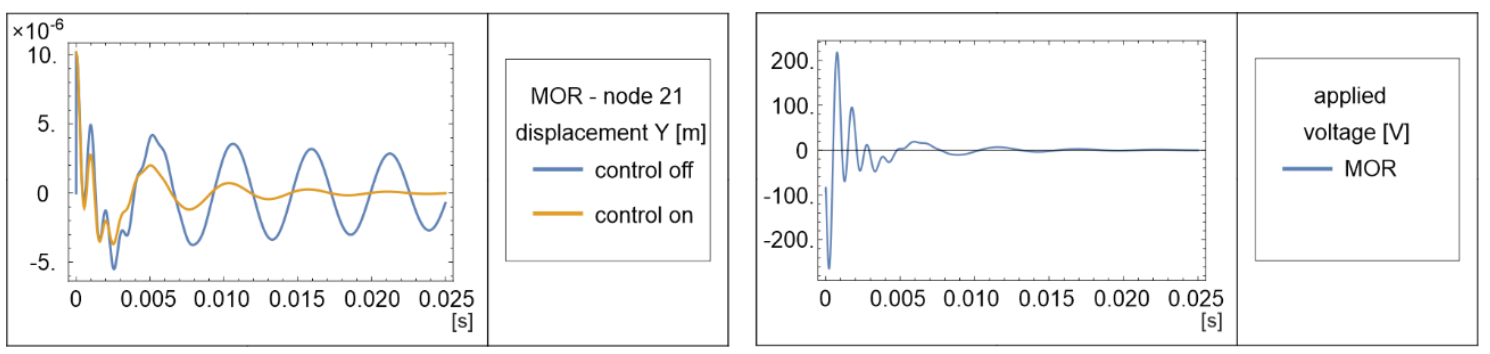

Fig. 14 Comparison of the system response, blue line is response of the system without control and brown line for the system with control - left, the electric potential on the actuators generated from the linear quadratic controller - right.

The electric potential on the piezoelectric actuators generated from the linear quadratic controller was determined - time variation of electric potential is shown in Fig. 14 right. This voltage was applied as a load to the full FEM model and a transient FEM analysis was performed. A comparison of the results from the FEM model and the MOR model when control is considered is shown in Fig. 15, where the blue line represents the displacement of node 21 from the FEM analysis and the brown rings from the MOR analysis.

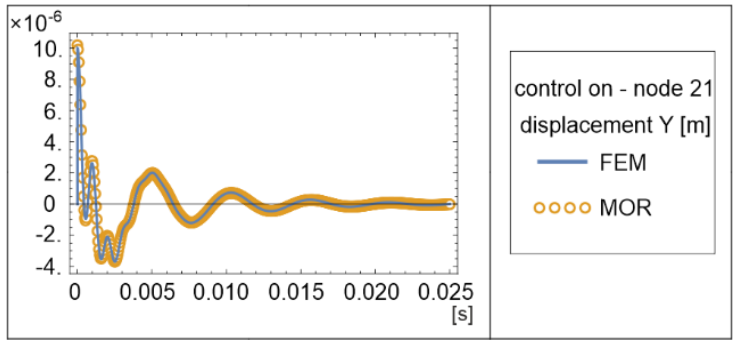

Fig. 15. Response of system with control computed by FEM model - blue line and response of system with control computed by MOR model - brown rings.

As can be seen from obtained results, the MOR model of investigated system very faithfully describes the behaviour of the system, where the FEM model was used as a reference model.

\subsection{FGM beam structure with piezoelectric layers}

\section{Analysed system}

The analysed system is shown in Fig. 16 left. It consists of 3 beams: beam 1 and beam 2 are made of functionally graded material with variation of material properties along the length and height of the beam, beam 3 has constant material properties. A piezoelectric actuator is located on the beam 1 and a piezoelectric sensor is placed on the beam 2 . 


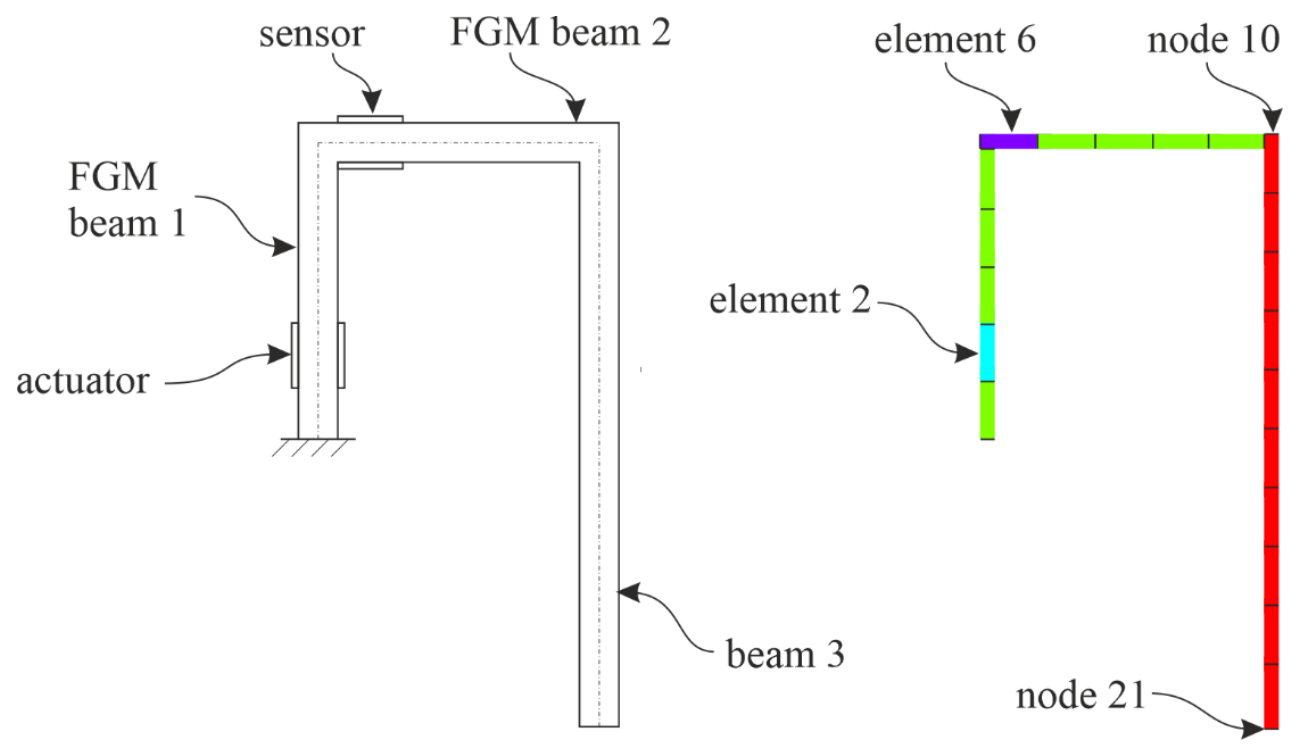

Fig. 16 Piezoelectric structure with actuator and sensor - left, discretized FEM model - right.

Geometry parameters of beams are:

- cross-section - all beams have height and depth of cross-section $0.01 \mathrm{~m}$, height of piezoelectric layers is $0.001 \mathrm{~m}$ and its depth is $0.01 \mathrm{~m}$

- length - beams 1 and 2 have length $0.05 \mathrm{~m}$ and beam 3 has length $0.1 \mathrm{~m}$, length of piezoelectric layers is $0.01 \mathrm{~m}$

Material parameters of beams are:

- beams 1 and 2 are made from FGM - homogenized material properties of FGM core without piezoelectric layers as well as FGM core with piezoelectric layers were defined in section 6.1

- beam 3 has constant material properties: $E=319.4 \mathrm{GPa}, \rho=13989.6 \mathrm{~kg} / \mathrm{m}^{3}$

- damping of structure is considered as Rayleigh damping with mass and stiffness constants $1 \times 10^{-5} \mathrm{~s}^{-1}$ and $1 \times 10^{-5} \mathrm{~s}$, respectively

- the inner electrodes of piezoelectric layers are grounded.

The goal is to perform a FEM transient analysis of the system under defined initial conditions, to create an MOR model using the modal properties of the system, to compare the results from FEM and MOR models, to design of the linear quadratic regulator (LQR) and compare the response of the system without control and with control.

\section{Analysis of FEM model}

The initial state of the analysed structure is defined by the static deformation of the structure, when the horizontal displacement of the free end of beam structure is prescribed node 21 (see Fig. 16), the displacement has value $5 \times 10^{-6} \mathrm{~m}$. Free vibration of structure is investigated.

The discretized FEM model is shown in Fig. 16 right. The response of the system - the vertical displacement of nodes 21 and 10 and electric charge on element 6 is shown in Fig. 17. 

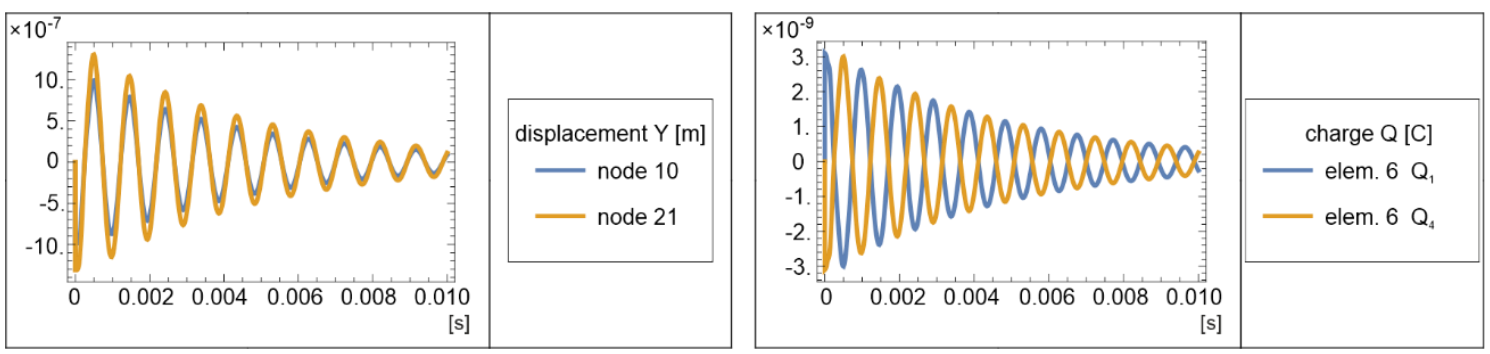

Fig. 17 The response of the system, the vertical displacement of nodes 21 and $10-$ left, electric charge on element 6 - right.

The goal of the modal analysis is to obtain the first four bending eigenfrequencies and the corresponding eigenmodes of the analysed beam structure. These eigenfrequencies and eigenmodes are subsequently used both in the creation of a reduced model of the system and in the definition of the modal initial state of the system. The first four eigenshapes are shown in Fig. 18 and corresponding eigenfrequencies are $1040.24 \mathrm{~Hz}, 4251.05 \mathrm{~Hz}, 7256.15 \mathrm{~Hz}$ and $17477.4 \mathrm{~Hz}$.

\begin{tabular}{|c|c|c|c|c|c|}
\hline $1040.24 \mathrm{~Hz}$ & 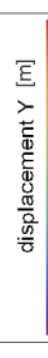 & $\begin{array}{l}+0 \\
-518.7 \times 10^{-6} \\
-1.037 \times 10^{-3} \\
-1.556 \times 10^{-3} \\
-2.075 \times 10^{-3} \\
-2.593 \times 10^{-3} \\
-3.112 \times 10^{-3} \\
-3.631 \times 10^{-3} \\
-4.149 \times 10^{-3} \\
-4.668 \times 10^{-3}\end{array}$ & $4251.05 \mathrm{~Hz}$ & 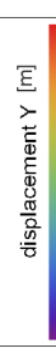 & $\begin{array}{l}+5.206 \times 10^{-3} \\
+4.628 \times 10^{-3} \\
+4.049 \times 10^{-3} \\
+3.471 \times 10^{-3} \\
+2.892 \times 10^{-3} \\
+2.314 \times 10^{-3} \\
+1.735 \times 10^{-3} \\
+1.157 \times 10^{-3} \\
+578.5 \times 10^{-6} \\
+0\end{array}$ \\
\hline $7256.15 \mathrm{~Hz}$ & 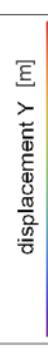 & $\begin{array}{l}+0 \\
-133.7 \times 10^{-6} \\
-267.5 \times 10^{-6} \\
-401.2 \times 10^{-6} \\
-534.9 \times 10^{-6} \\
-668.7 \times 10^{-6} \\
-802.4 \times 10^{-6} \\
-936.1 \times 10^{-6} \\
-1.07 \times 10^{-3} \\
-1.204 \times 10^{-3}\end{array}$ & $17477.4 \mathrm{~Hz}$ & 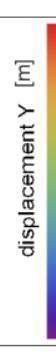 & $\begin{array}{l}+6.593 \times 10^{-3} \\
+5.532 \times 10^{-3} \\
+4.471 \times 10^{-3} \\
+3.41 \times 10^{-3} \\
+2.349 \times 10^{-3} \\
+1.288 \times 10^{-3} \\
+227.1 \times 10^{-6} \\
-833.9 \times 10^{-6} \\
-1.895 \times 10^{-3} \\
-2.956 \times 10^{-3}\end{array}$ \\
\hline
\end{tabular}

Fig. 18 The first four bending eigenmodes.

\section{Analysis of MOR model}

A reduced model was created using the modal truncation method, considering the first four eigenshapes of the structure shown in Fig. 18 and their corresponding natural frequencies. The created reduced state-space model was analysed in program Mathematica using the StateResponse and OutputResponse commands, which simulate state quantities and output quantities of defined state-space mode, respectively. The obtained state and output quantities from the reduced state-space model were compared with the results obtained from the FEM model - Fig. 19, where FEM results are shown by a blue line, and MOR results by a brown ring. Fig. 19 left shows the vertical displacement of the node 21 and Fig. 19 right shows the electric charge on the piezoelectric sensor - element 6. 

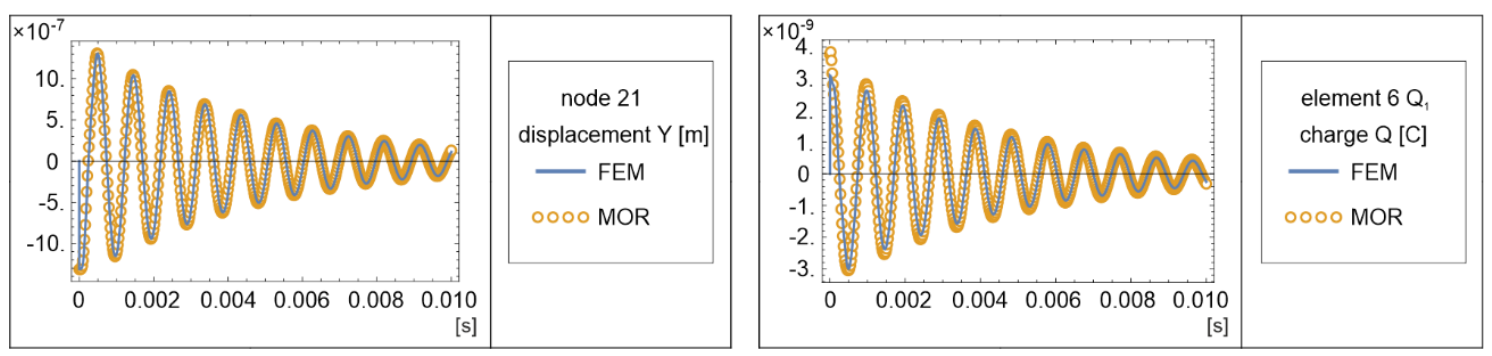

Fig. 19 Results calculated by FEM model (blue line) and by MOR model (brown rings), vertical displacement of free end - left, electric charge on sensor - right.

As can be seen from these results, the displacement as well as the electric charge obtained from the MOR simulation correspond very well with quantities obtained from the FEM analysis.

\section{Control of MOR model}

For the analysed system, a linear quadratic regulator was proposed with the smae state weight and the same input weight as were used in previous example. The output of gain matrix of regulator is the electrical voltage for the piezoelectric actuator and the input of controller is the full state of the model. The proposed LQR controller was connected with reduced state-space model and the final closed loop system was analysed. The initial state of closed loop system was defined in the same way as in the transient FEM analysis. A comparison of a deformation of a system represented by vertical displacement of node 21 without control and a system with control is shown in Fig. 20 left, Fig. 20 right shows the electrical voltage that is applied to the piezoelectric actuator as controller output.
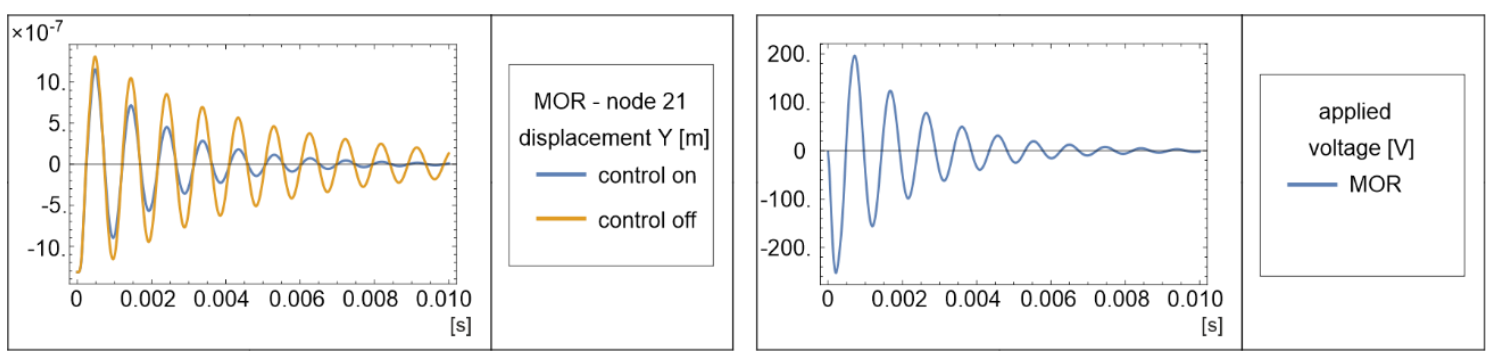

Fig. 20 A comparison of a deformation of a system represented by vertical displacement of node 21 without control (brown line) and a system with control (blue line) - left, the electrical voltage applied to the piezoelectric actuator - right.

\section{Conclusions}

The paper presents beam finite element with piezoelectric layers, where core of the beam is made of FGM materials. Such combination of materials is very attractive for mechatronic applications because material composition of FGM core can be optimized for design stress state and deformation can be controlled by voltages on electrodes. The derived equations were implemented in the FEM program MultiFEM. Subsequently, FEM model was reduced using the modal truncation method, which is one of the MOR methods. The created MOR model is then used to design the LQR controller. The MOR model with feedback with the LQR controller is also investigated and the results are compared with the FEM model with the same electrical load on the piezoelectric actuators.

\section{ACKNOWLEDGEMENT}

This work was supported by the Slovak Grant Agency: VEGA 1/0081/18, KEGA 011STU4/2020 and APVV-19-0406. 


\section{REFERENCES}

[1] Bolton, W. "Mechatronics, Electronic Control Systems in Mechanical and Electrical Engineering", 6th edition, Pearson, 2015. ISBN: 9781292076683

[2] Schwartz, M. "Encyclopedia of Smart Materials", John Wiley \& Sons, Inc., 1st edition, 2002. ISBN: 0471177806.

[3] Arnau, A. "Piezoelectric Transducers and Applications", Springer, 2nd edition. 2008. ISBN: 9783540775072

[4] Dorf, R. C., Bishop, R. H. "Modern Control Systems", Pearson, 13th edition. 2016. ISBN: 0134407628

[5] Burnett, D.S. "Finite Element Analysis: From Concepts to Applications", Addison Wesley Publishing Company, 1st edition, 1987. ASIN: B01FIWOG9A

[6] Besselink, B. et al. "A Comparison of Model Reduction Techniques from Structural Dynamics", In Numerical Mathematics and Systems and Control, Journal of Sound and Vibration 332, pp. 4403 - 4422, 2013.

[7] Lewis, F. L., Varabie, D., Syrmos, V. L. "Optimal Control”, John Wiley and Sons, Third edition, 2012. ISBN: 9780470633496

[8] Mahamood, R. M., Akinlabi, E.T. "Functionally Graded Materials", Springer International Publishing, 2017. ISBN: 9783319537566.

[9] Murín J., Hrabovský J., Aminbaghai M., Kutiš V., Paulech J., Kugler S. "Extension of the FGM Beam Finite Element by Warping Torsion", Strojnícky časopis - Journal of Mechanical Engineering 69 (2), pp. 57 - 76, 2019. DOI: 10.2478/scjme-2019-0017

[10] Wolfram Research, Inc. Mathematica, Champaign, Illinois, Version 12.3. 2021.

[11] Kutiš, Murín, J., Belák, R., and Paulech J. "Beam Element with Spatial Variation of Material Properties for Multiphysics Analysis of Functionally Graded Materials", Computers and Structures 89, pp. 1192 - 1205, 2011.

[12] Nye, J., F. "Physical Properties of Crystals: Their Representation by Tensors and Matrices”, Oxford University Press, 1985. ISBN: 0198511655

[13] Kutiš, V., Paulech J., Murín J., Gálik, G. “Analysis of Piezoelectric Beams for Smart Structures", In: 24th International Conference on Applied Physics of Condensed Matter, June 20 - 22, 2018 - Štrbské Pleso, Slovakia, 2018.

[14] Piefort, V. "Finite Element Modeling of Piezoelectric Structures", PhD Thesis. Universite Libre de Bruxelles, 2001.

[15] Friedland, B. "Control System Design: An Introduction to State-Space Methods", Dover Publications, Illustrated edition, 2005. ISBN: 0486442780 